\section{Smoke gets in your eyes}

Shamira Perera ${ }^{1,2,3,4}$

${ }^{1}$ Glaucoma Service, Singapore National Eye Centre, Singapore; ${ }^{2}$ Bioengineering and Devices, Singapore Eye Research Institute, Singapore; ${ }^{3}$ Clinical and Surgical Innovation, Ophthalmology \& Visual Sciences Academic Clinical Programme, Duke-NUS Graduate Medical School, Singapore; "Medical Technology Office, SingHealth Research, Singapore

In this inaugural issue of Malaysian Journal of Ophthalmology, Nurul-Laila Salim and associates describe their case control study to determine the association between cigarette smoking and primary angle-closure glaucoma (PACG) in Malay patients. The authors should be commended on their efforts in investigating a regionally relevant and visually destructive disease and searching for presently elusive modifiable risk factors. Tight definitions of disease, a rigorous questionnaire process, and a well-delineated ethnic population are the strengths of this study, which continues the trend for high quality PACG research from the region.

Glaucoma includes a group of disorders characterized by progressive damage to the optic nerve associated with characteristic loss of the field of vision. It is the leading cause of irreversible vision impairment worldwide, with PACG a major subtype of glaucoma and cause of blindness, particularly in Asia. PACG is estimated to affect $>20$ million people. The greatest challenge in preventing glaucoma blindness is identifying which individuals with PACG are at greatest risk of deterioration and development of advanced disease and what can be done to arrest disease progression. Unfortunately, the only well accepted modifiable risk factor at present is elevated intraocular pressure; this itself took many years to establish.

Although inconclusive, cigarette smoking has been thought to increase the risk of primary open-angle glaucoma (POAG), which is the more intensively studied type of glaucoma. However, the exact nature of this biologically plausible relationship is unclear, as the recent systematic review explains. ${ }^{1}$ Some suggested that this association was related to the number of pack-years, as opposed to former smokers or passive smokers, but the dose-response effect is not clear. In contrast, PACG has been far less scrutinized and really only in-depth in regions where it is impossible to ignore, such as South East Asia. To see such a study on the Malaysian Malay population is a very much welcome development, as the results could be extrapolated to some extent to all Malays in the region, which number a sizeable population. 
The type of investigation being used to its full effect here is a case control study, the stalwart of epidemiological studies to discover associations and their strength. The conclusions here pave the way toward more directed exploration to make the findings more meaningful, as again, there appears to be no logical dose-response relationship found, this time between smoking and PACG. Future cohort studies can examine causation and at what time point and intensity smoking can have its greatest impact so that fundamental research can be directed towards the mechanisms involved.

In Malaysia, smoking still has some social taboo attached, and yet its prevalence is surprisingly high and static (around $46 \%$ in males) despite sustained anti-smoking measures. ${ }^{2}$ Some inroads have been made to decrease the prevalence of smoking, including curtailing advertising, designation of smoke-free areas, restructuring of tobacco taxes, anti-smoking campaigns, and the introduction of smoking cessation services at health clinics. This study adds to the weight of evidence behind the harms of smoking (beyond cancer and cardiovascular disease) and could be used to fuel further initiatives in public policy.

Based on the present study, ex-smoker and active smoker groups were not found to be significantly associated with PACG. However, there was a significant association between passive smokers and PACG in Malay patients residing in Malaysia. The importance of this novel finding resides in opening up the possibility to control the disease by modifying behaviors, which could be mediated via ramping up existing public health efforts against smoking in general.

Biometric and demographic risk factors for PACG have been well investigated and probably overshadow the effects of passive smoking in this population. Nevertheless, the increased risk of PACG to passive smokers is impressively large and an association such as this has not been seen in POAG to date. This novel finding is noteworthy, as many other studies have not considered the aspect of passive smoking, which might be the most amenable to intervention by carefully targeting families and workspaces with more information. The difficulty arises though, as the amount of passive smoking is difficult to quantify.

A separate, but related issue has grown since this study collected data: the controversial practice of vaping. A study from Klang in Malaysia ${ }^{3}$ found that adult vapers perceived e-cigarettes as less toxic and healthier than cigarettes. However, whilst they are portrayed as less harmful and containing fewer contaminants, the chemicals contained in e-cigarettes are yet to be fully characterised and assessed for risk. Malaysia's 2019 ban on smoking at restaurants and eateries includes e-cigarettes if they contain nicotine, but how this will affect the passive smoking landscape remains to be seen. 


\section{References}

1. Jain V, Jain M, Abdull MM, Bastawrous A. The association between cigarette smoking and primary open-angle glaucoma: a systematic review. Int Ophthalmol. 2017;37(1):291-301

2. Lim HK, Ghazali SM, Kee CC, et al. Epidemiology of smoking among Malaysian adult males: prevalence and associated factors. BMC Public Health. 2013;13:8.

3. Zainol Abidin N, Abidin EZ, Zulkifli A, et al. Vaping topography and reasons of use among adults in Klang Valley, Malaysia. Asian Pac J Cancer Prev. 2018;19(2):457-462. doi:10.22034/APJCP.2018.19.2.457
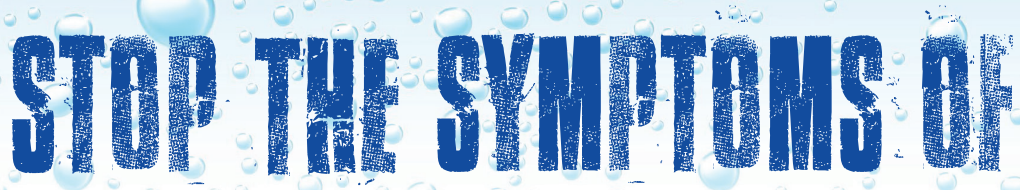

Refresh
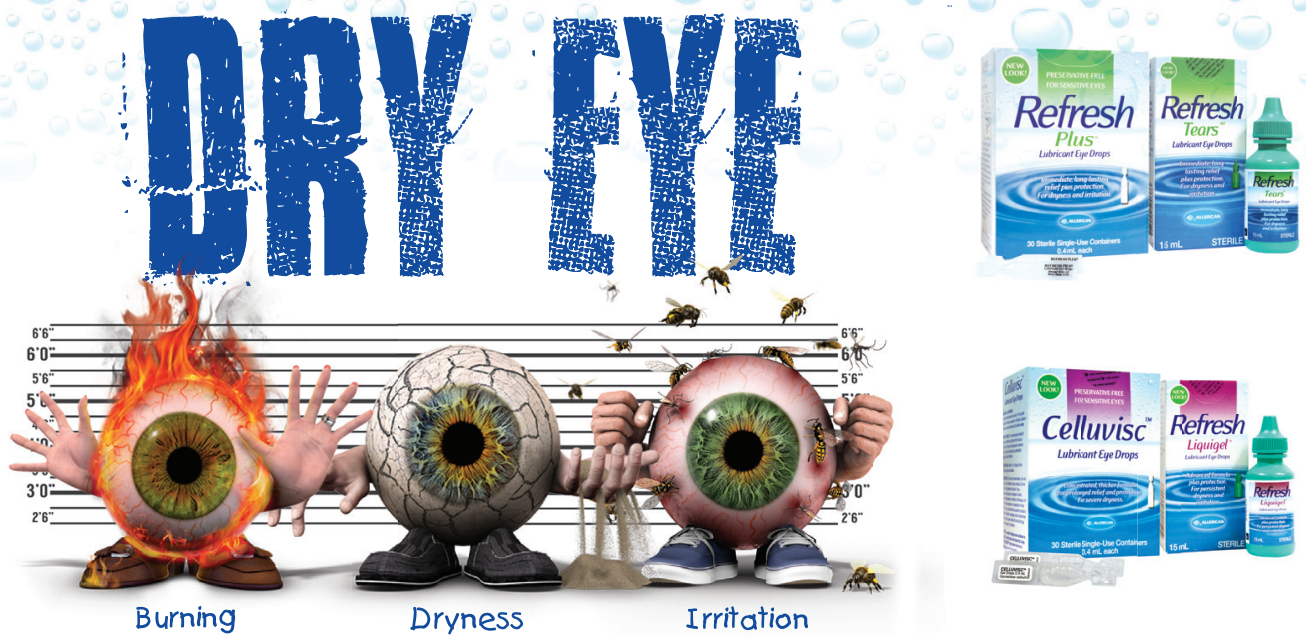

Burning

Irritation

Refresh range of lubricating eye drops keeps your eye healthy and away from these dry eye related conditions. 